PALEO

Revue d'archéologie préhistorique

$24 \mid 2013$

Varia

\title{
Une vie consacrée à la science du Sahara et de son passé
}

Berny Sèbe

\section{(2) OpenEdition}

Journals

Édition électronique

URL : http://journals.openedition.org/paleo/2697

DOI : $10.4000 /$ paleo.2697

ISSN : 2101-0420

Éditeur

SAMRA

\section{Édition imprimée}

Date de publication : 15 décembre 2013

Pagination : $279-280$

ISSN : 1145-3370

\section{Référence électronique}

Berny Sèbe, «Une vie consacrée à la science du Sahara et de son passé », PALEO [En ligne], 24 | 2013,

mis en ligne le 17 avril 2014, consulté le 07 juillet 2020. URL : http://journals.openedition.org/paleo/ 2697 ; DOI : https://doi.org/10.4000/paleo.2697

Ce document a été généré automatiquement le 7 juillet 2020

\section{(c) (i) () $\Theta$}

PALEO est mis à disposition selon les termes de la licence Creative Commons Attribution - Pas d'Utilisation Commerciale - Pas de Modification 4.0 International. 


\section{Une vie consacrée à la science du Sahara et de son passé}

\section{Berny Sèbe}

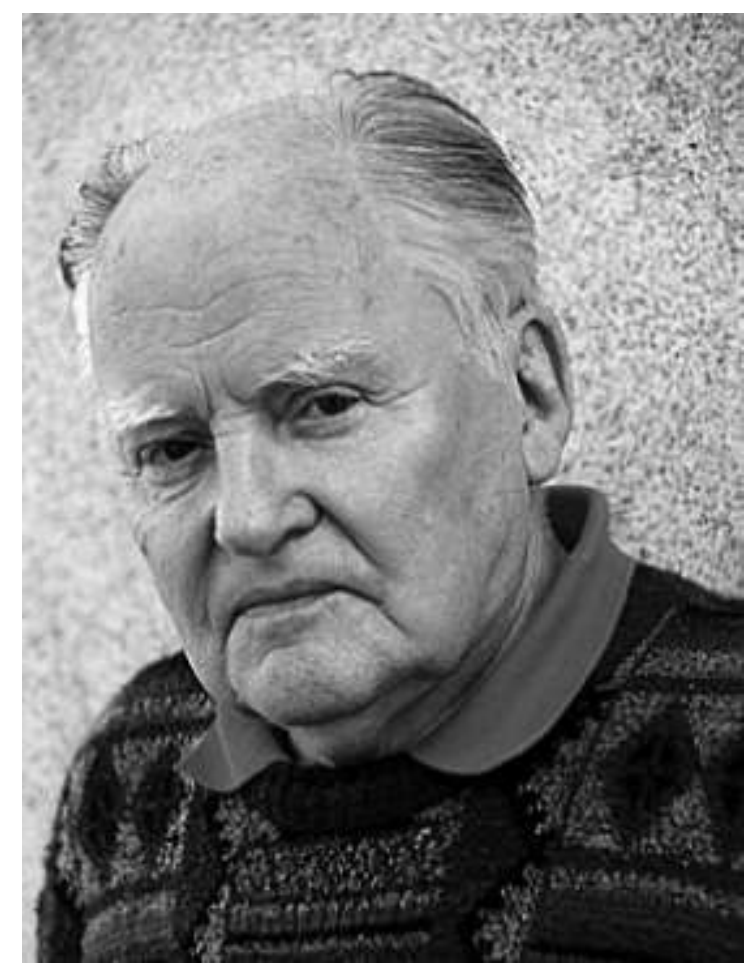

1 Né en 1916, Henri-Jean Hugot est le dernier représentant de l'élite des savants pluridisciplinaires du Sahara encore parmi nous. A l'instar des Théodore Monod ou des Henri Lhote, qui nous ont quittés dans les deux dernières décennies, il s'est intéressé au cours de sa longue carrière scientifique à de nombreux aspects du passé des grands espaces sahariens, offrant des perspectives pionnières qui ont permis de notables avancées dans la compréhension des populations préhistoriques du Sahara. 
2 Après avoir étudié l'ethnologie et la paléontologie, Henri-Jean Hugot débute sa carrière de préhistorien avec plusieurs articles scientifiques fondés sur des fouilles qu'il a menées lui-même, notamment dans le Tidikelt algérien, «au pied du batèn du Tademaït, qui est à la fois un enfer torride et un paradis pour le préhistorien », comme le remarquait Lionel Balout ${ }^{1}$. Ces recherches conduites dans l'immédiat après-guerre contribueront à ouvrir de nombreuses perspectives pour la préhistoire saharienne, notamment en conceptualisant une possible période pré-néolithique dans la région. Au département de préhistoire saharienne du musée de préhistoire et d'ethnographie du Bardo à Alger, où il est stagiaire puis attaché de recherche au CNRS entre 1956 et 1962, Henri-Jean Hugot travaille avec les plus grands préhistoriens de l'Afrique du Nord, tels Maurice Reygasse, Gabriel Camps ou Lionel Balout. Il participe aux deux missions Berliet Ténéré Tchad (novembre 1959-janvier 1960 ; octobre-décembre 1960), grandes expéditions scientifiques de Djanet à Fort-Lamy (aujourd'hui N'Djaména) dirigées par l'industriel automobile passionné du Sahara, Maurice Berliet. Alors qu'un autre grand Saharien, littéraire celui-là, Roger Frison Roche, se charge de raconter la mission au grand public grâce à un livre publié chez Arthaud, Henri-Jean Hugot se charge de la direction scientifique d'un imposant volume de près de quatre cents pages, luxueusement publié par les Editions des Arts et Métiers Graphiques, réunissant la somme des observations morphologiques, géologiques, préhistoriques et paléoclimatiques effectuées sur le trajet des missions. Accompagné d'un volume annexe réunissant les documents scientifiques, l'imposant ouvrage Missions Berliet Ténéré Tchad est resté jusqu'à nos jours une référence incontournable pour quiconque s'intéresse à la préhistoire et aux réalités physiques du Sahara ${ }^{2}$. La publication de ces volumes est suivie de peu par celle de ses Recherches préhistoriques dans l'Ahaggar Nord-occidental, 1950-1957, véritable inventaire de l'état des connaissances sur le Paléolithique inférieur, l'Atérien et le Néolithique dans la région du nord du Hoggar (essentiellement l'Immidir). Henri-Jean Hugot y remarquait déjà ce qui allait apparaître comme un leitmotiv dans sa relation au désert : "Le Sahara en lui-même est une autre chance pour qui sait le voir comme le plus merveilleux livre d'images qui ait été gardé au préhistorien par la sage et prévoyante nature. ${ }^{3}$ C'est également au cours de cette période qu'il tente d'établir dans son Essai sur les armatures de pointes de flèches du Sahara une typologie de l'un des artefacts les plus répandus sur l'ensemble des territoires sahariens, et qui fut un élément vital pour la survie de civilisations de chasseurs. Il y démontre notamment la richesse et la diversité des formes de cet outil au Sahara ${ }^{4}$.

3 Après l'indépendance de l'Algérie, la carrière de Henri-Jean Hugot se déplace vers l'Afrique de l'Ouest : à Dakar, il prend la suite de Raymond Mauny comme directeur du département d'archéologie et de préhistoire à l'Institut français (devenu «fondamental» en 1966) d'Afrique noire, l'IFAN, de 1963 à 1969. C'est là qu'il se rapproche d'une région qui deviendra l'un de ses terrains favoris, le Dhar Tichitt, où il mènera de nombreux chantiers de fouilles à partir de $1966^{5}$. Ses observations dans le Sud-Est mauritanien fourniront, aux côtés de celles menées dans le Sahara algérien, une grande partie de la matière offerte dans l'une des ses œuvres majeures, l'ouvrage Le Sahara avant le désert, qui présente une vision synthétique des populations sahariennes aux temps préhistoriques. Ce livre pionnier tentait de tirer le meilleur d'un siècle de fouilles archéologiques au Sahara, brossant un portrait humain de la région depuis la période pré-Néanderthalienne jusqu'aux époques lybico-berbères, et tentant de distinguer par là-même les diverses influences qui ont marqué le Néolithique saharien (en particulier les traditions capsienne et soudanienne, qui ont laissé, d'après Henri- 
Jean Hugot, « des civilisations matérielles différentes, voire opposées »). Plus généralement, il $\mathrm{y}$ avance avec justesse que "la finalité de la Préhistoire c'est en définitive de rendre à l'homme la connaissance totale de son passé $»^{6}$.

4 Les années 1970 voient Henri-Jean Hugot revenir en France métropolitaine, où il exerce notamment le rôle de vice-directeur du Museum national d'histoire naturelle et de sous-directeur au Musée de l'Homme. Il poursuit en parallèle sa production scientifique, continuant notamment son travail sur le terrain mauritanien. En tant que Professeur des universités, Henri-Jean Hugot dirige également de nombreuses thèses, ce qui lui permet de faire fructifier l'héritage intellectuel qu'il laisse à la profession. Il co-dirige en 1981 un volume de mélanges offerts au grand préhistorien Lionel Balout (1907-1992), preuve de sa place éminente dans la préhistoire française de l'époque ${ }^{7}$. En parallèle à ses nombreuses activités scientifiques et administratives, Henri-Jean Hugot a aussi pris le temps de communiquer ses résultats au grand public. Il a ainsi écrit les textes de plusieurs ouvrages photographiques, tels Les gens du matin, dix mille ans d'art et d'histoire (1976), Sahara toujours recommencé (1979)8), Sahara art rupestre (1999) ou Tibesti, Sahara interdit (2005). Il a également produit plusieurs ouvrages de vulgarisation préhistorique pour le public scolaire en France et en Afrique, et dirigé le programme de préhistoire pour l'enseignement primaire en Afrique francophone et à Madagascar.

Henri-Jean Hugot est correspondant de la 5e section de l'Académie des Sciences d'Outre-Mer, et reste intellectuellement actif, faisant face avec détermination à une santé fragile et au choc représenté par la perte de son épouse Simone en juillet 2006. Il est soutenu en cela par ses nombreux enfants, unis par un précieux sentiment d'appartenance familiale.

\section{BIBLIOGRAPHIE}

B. SEBE - Henri-Jean Hugot - Une vie consacrée à la science du Sahara et de son passé

\section{NOTES}

1. H.-J. Hugot, « Du Caspien au Tidikelt », Actes du IIe congrès P.P.E.Q., 1955, p. 601-3 ; « Un gisement de pebble tools à Aoulef ", Trav. de l'I.R.S. (13), p.131-49; "Sur quelques coquilles du Mouydir (Sahara central) ", Lybica, 1957 (5), p. 21-31. La citation de Lionel Balout relative au Tidikelt provient de sa préface au volume de Henri-Jean Hugot, Le Sahara avant le désert, Toulouse, Editions des Hespérides, 1974, p. 11.

2. H.-J. Hugot (dir.), Mission Berliet Ténéré-Tchad. 9 nov. 1959-7 janv. 1960, 23 oct. 1960-9 déc. 1960, Paris, Arts et Métiers Graphiques, 1962 ; Mission Berliet Ténéré-Tchad. 9 nov. 1959-7 janv. 1960, 23 oct. 1960-9 déc. 1960 : documents scientifiques, Paris, Arts et Métiers Graphiques, 1962.

3. H.-J. Hugot, Recherches préhistoriques dans l'Ahaggar nord-occidental, Paris, Arts et Métiers Graphiques, 1963, p. 5.

4. H.-J. Hugot, «Essai sur les armatures de pointes de flèches du Sahara », Lybica, 1957 (5), p. 89-236. Réimpression : Calvisson, Editions Jacques Gandini, 1991. 
5. Voir par exemple, parmi ses nombreux travaux sur ce thème, H. J. Hugot, « Tichitt, réflexions sur le néolithique de l'Ouest africain », Revue de l'occident musulman et de la Méditerranée, 1972 (11), p. 31-33 ; H.-J. Hugot, « Où en sont les fouilles de Tichitt?», Miferma informations, 1973, p. 42-47; H.-J. Hugot et M.-P. Hugot-Buffet, Tichitt, Mauritanie du Sud-Est: étude de la ville actuelle, Paris, Ministère de la Coopération, 1977.

6. H.-J. Hugot, Le Sahara avant le désert, Toulouse, Editions des Hespérides, 1974. Les citations proviennent des pages 90 et 18 respectivement.

7. C. Roubet, H.-J. Hugot et G. Souville (dir.), Préhistoire africaine, mélanges offerts au Doyen Lionel Balout, Paris, A.D.P.F., 1981.

8. H.-J. Hugot et M. Bruggmann, Les gens du matin, Sahara : dix mille ans d'art et d'histoire, Paris, La Bibliothèque des Arts, 1976 ; H.-J. Hugot, J.-M. Durou et J. Jaffre, Sahara toujours recommencé, Paris, Hachette, 1978 ; H.-J. Hugot et M. Bruggmann, Sahara art rupestre, Paris, Les éditions de l'Amateur, 1999 ; H.-J. Hugot, P. Frey, M. Bruggmann, R. Depardon, A. et B. Sèbe, Tibesti, Sahara interdit, Vidauban, Alain Sèbe Images, 2005.

\section{AUTEUR}

\section{BERNY SÈBE}

Université de Birmingham (Royaume-Uni) 